\title{
Two-dimension nonlinear optimization method for parameter inversion of elliptic equation
}

\author{
QIAN Yun ${ }^{1}$ \\ Department of Mathematics \\ Chaohu University \\ Chaohu ,China \\ e-mail: qianyun@chu.edu.cn
}

\author{
Liu Xiangguo ${ }^{2}$ \\ Department of Mathematics \\ Chaohu University \\ Chaohu ,China \\ e-mail: liuxingguo888@163.com
}

\begin{abstract}
The paper researches the parametric inversion of the two-dimensional ellipse equation by means of non-linear optimization method, draw a Numerical Solution for such inverse problem. It is shown by numerical simulations that the method is feasible and effective.
\end{abstract}

Keywords- parameter; inverse problem; non-linear optimization method

\section{I.INTRODUCTION}

Elliptic partial differential equations in many practical problems have a very wide range of application, such as the proliferation of mass concentration of pollutants in the atmosphere and water, sea water salinity and temperature diffusion, fluid flow and heat transfer, the spread of pollutant transport, electro-chemical reaction, the proliferation and transport of pollutants, electro-chemical reaction, such problems have become issues of concern to many academics. Parameter inversion is an important component of convection-diffusion model; its correctness directly affects the reliability of the model.For this reason, many workers have done a lot of research work at the methods of parametric inversion in the model. The former Soviet Union scholar Tikhonov put forward the Regularization methods for solving ill-posed problems, effectively solved the problems. Our famous pioneers of computational mathematics, the late Academicians of CAS , professor Feng Kang had strongly advocated the study on the numerical solution of an inverse problem as early as the 1980s, which has had a profound impact on the research and application of inverse problems of mathematical physics in China ${ }^{[1,2]}$.

In recent years,?with the revision and development of Mr.Chen Yongmin and other Chinese scholars, they proposed the technology of a generalized pulse spectrum ${ }^{33}$ and apply the method to solve inverse problems for a number of areas. But for the study of inverse problems of partial differential equations, there is a significant progress from theory to practical solution. They have made a lot of effective numerical methods: Such as homotopy method ${ }^{[4]}$, inverse scattering inversion, methods of Monte Carlo and so on. Those methods are mainly applied to solve inverse

Anhui university provincial natural science research project (KJ2012A205) problems for partial differential equations. But for inverse problems for 2-dimensional partial differential equation , the study is much less. This paper has born fruits on the research of the parameter inversion problem of twodimensional elliptic partial differential equations with the method of nonlinear optimization ${ }^{[5,6]}$.

\section{II.MATHEMATICAL MODEL OF INVERSE PROBLEM OF TWO- DIMENSIONAL ELLIPTIC PARTIAL DIFFERENTIAL EQUATIONS WITH COEFFICIENTS}

Consider the problem of two-dimensional elliptic partial differential equations boundary value

$$
\begin{aligned}
& {\left[-\frac{\partial}{\partial x}\left[a(x, y) \frac{\partial u}{\partial x}\right]-\frac{\partial}{\partial y}\left[a(x, y) \frac{\partial u}{\partial y}\right]\right.} \\
& +\frac{\partial}{\partial x}\left(b_{1}(x, y) u\right)+\frac{\partial}{\partial y}\left(b_{2}(x, y) u\right)+c(x, y) u=f(x, y) \quad(x, y) \in \Omega
\end{aligned}
$$

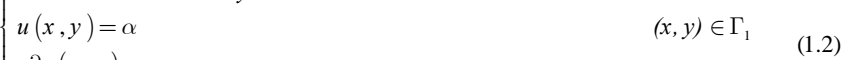

$$
\begin{aligned}
& a \frac{\partial u(x, y)}{\partial n}+\sigma u(x, y)=\beta \quad(x, y) \in \Gamma_{2}
\end{aligned}
$$

Of which $\Gamma_{1} \cap \Gamma_{2}=\theta, \Gamma_{1} \cup \Gamma_{2}=\partial \Omega$ is the Regional boundaries of $\Omega, \theta$ is empty set . when $a(x, y)$, $b_{1}(x, y), b_{2}(x, y), c(x, y), f(x, y), \sigma$, $\beta$ are Given the known function, solving $u(x, y)$ forms the value problems of Steady-state convection-diffusion equations with boundary. They have different physical meaning in many practical applications: $a(x, y)$ represents durchgriff, $\left(b_{1}(x, y), b_{2}(x, y)\right)$ represents potential function, $f(x, y)$ represents source function . But when $u(x, y), f(x, y), b_{1}(x, y), b_{2}(x, y)$, $c(x, y), \sigma, \alpha, \beta$ are Given the known function, and the need solving is parameter, which forms the inversion problem of Two-dimensional elliptic boundary value, at this 

to the boundary

$$
\begin{aligned}
& u(x, y)=g(x, y) \\
& (x, y) \in \Gamma_{2}
\end{aligned}
$$

$$
a \frac{\partial u(x, y)}{\partial n}+\sigma u(x, y)=q(x, y),(x, y) \in \Gamma_{1}
$$

Condition (1.4) or (1.5) is called the complement of the inverse problem solution conditions (also known as the calibration conditions).

\section{NONLINEAR OPTIMIZATION OF PARAMETER INVERSE PROBLEM OF TWO-DIMENSIONAL ELLIPTIC PARTIAL DIFFERENTIAL EQUATIONS}

For equation (1.1), functions $b_{1}(x, y), b_{2}(x, y)$, $c(x, y), f(x, y)$ are known, if given $a(x, y)$ from equation (1.1) and already known boundary Condition ( 1.2 ) and ( 1.3 ) we get $u(x, y)$, do Bivariate polynomial approximation to $a(x, y)$ time, there is a need to supply Additional measurement data

In complementing the discrete access point solution conditions and numerical solution to be calculated according to equations, so ( 2.1 ) or ( 2.2 ) can be converted to Discrete optimization problems

$\left.J_{1}\left(\alpha_{0}, \alpha_{1}, \cdots ; \alpha_{n}\right)=\min _{\left(\alpha_{1}, \alpha_{1}, \cdots, \alpha_{n}\right) \in R^{n}} \sum_{i=1}^{m} \sum_{j=1}^{l} u\left(\alpha_{b}, \alpha_{1}, \cdots ; \alpha_{n}, x_{i}, y_{j}\right)-g_{1}\left(x_{i}, y_{j}\right)\right)$

Or

$J_{2}\left(\alpha_{0}, \alpha_{1}, \cdots, \alpha_{n}\right)=\min _{\left(\alpha_{i}, \alpha_{1}, \cdots, \alpha_{n}\right) \in R^{n}} \sum_{i=1}^{m} \sum_{j=1}^{l}\left(\vec{n} \cdot u\left(\alpha_{0}, \alpha_{1}, \cdots, \alpha_{n}, x_{i}, y_{j}\right)-g_{2}\left(x_{i}, y_{j}\right)\right)$

If (2.3) and (2.4) as GA fitness functions, adjust parameter (2.3) and (2.4) to reach the minimum, so you can get the optimal approximation solutions.

When using the above algorithm, each computation to solve direct problem, and accuracy of direct problem $u(x, y)$ has a direct bearing on precision in inverse problem of solving $a(x, y)$. so in parametric inversion , with what method to solve $u(x, y)$ is also very important, this article using finite element method for solving $u(x, y)$

\section{NUMERICAL EXAMPLE}

$a(x, y)=\alpha_{0}+\alpha_{1} x+\alpha_{2} y+\alpha_{3} x y+\alpha_{4} x^{2}+\alpha_{5} y^{2}+\cdots$,

To test the effectiveness of the methods above, using the $\alpha_{i} \in R,(i=0,1,2, \cdots)$. So determining $a(x, y)$ equals determining $\alpha_{0}, \alpha_{\mathrm{g}}, \cdots ; \alpha_{h} \cdot \cdots$, Finite approximation $a(x, y)$.

It into equation (1.1) can determine the boundary conditions $u(x, y)$.Record

$$
u(x, y)=u\left(\alpha_{0}, \alpha_{1}, \alpha_{2}, \cdots, \alpha_{n}, x, y\right)
$$

Use of additional boundary conditions(1.4)or(1.5)the inverse problem can be transformed into the following noulinear optimization problem:

$$
J_{1}\left(\alpha_{1}, \alpha_{1}, \cdots ; \alpha_{n}\right)=\operatorname{minmax}_{\substack{\left(\alpha_{0}, \alpha_{1}, \cdots ; \alpha_{n}\right) \in R^{n} \\(x, y) \in \Omega_{\xi}}}\left\|u\left(\alpha_{1}, \alpha_{2}, \cdots ; \alpha_{n}, x, y\right)-g_{1}(x, y)\right\|_{2}
$$

Or

$$
J_{2}\left(\alpha_{1}, \alpha_{1}, \cdots ; \alpha_{n}\right)=\operatorname{minmax}_{\substack{\left(\alpha_{0}, \alpha_{1}, \cdots ; \alpha_{n}\right) \in R^{n} \\(x, y) \in \partial \delta_{\eta}}}\left\|\vec{n} \cdot u\left(\alpha_{1}, \alpha_{2}, \cdots ; \alpha_{n}, x, y\right)-g_{2}(x, y)\right\|_{2}
$$
above algorithm for numerical simulation program, and all are made on a p4-2.6G computer.

Example 1:

Consider the boundary value problem:

$$
\begin{cases}-\frac{\partial}{\partial x}\left[a(x, y) \frac{\partial u}{\partial x}\right]-\frac{\partial}{\partial y}\left[a(x, y) \frac{\partial u}{\partial y}\right] & \\ +\frac{\partial}{\partial x}\left(b_{1}(x, y) u\right)+\frac{\partial}{\partial y}\left(b_{2}(x, y) u\right)+c(x, y) u=f(x, y) & (x, y) \in \Omega \\ u(x, y)=0 & (x, y) \in \Gamma_{1} \\ \frac{\partial u(x, y)}{\partial n}=0 & (x, y) \in \Gamma_{2}\end{cases}
$$

Of which, $\Omega=(0,1) \times(0,1)$, $a(x, y)=1+x+2 y^{2}$,

$b_{1}=1+2 y, \quad b_{2}=x+1, c=x-y$; true solution: $\mathrm{u}=x y(1-x)(1-y), f(x, y)$ can be calculated. 
For example 1 , its Maximum inaccuracy, relative inaccuracy and Running time are in Graph 1

Graph 1 Calculation results

\begin{tabular}{|c|c|c|c|}
\hline $\mathrm{n}$ & $\begin{array}{c}\text { Maximum } \\
\text { inaccuracy }\end{array}$ & $\begin{array}{c}\text { relative } \\
\text { inaccuracy }\end{array}$ & Running time \\
\hline 16 & $1.0330 \times 10^{-4}$ & $1.4545 \times 10^{-3}$ & $0.1250(\mathrm{~S})$ \\
\hline 64 & $6.4866 \times 10^{-6}$ & $9.1111 \times 10^{-5}$ & $0.7340(\mathrm{~S})$ \\
\hline 256 & $4.0548 \times 10^{-7}$ & $5.6952 \times 10^{-6}$ & $13.5630(\mathrm{~S})$ \\
\hline
\end{tabular}

Note: $h$ is step index, $U$ is true solution, $u$ is numerical solution ,relative inaccuracy is $e 1=\frac{\|U-u\|_{2}}{\|U\|_{2}}$, Maximum inaccuracy is $\mathrm{d} 1=|U-u|, s$ is second.

\section{Example 2}

Considering Two-dimensional elliptic equations with variable coefficients

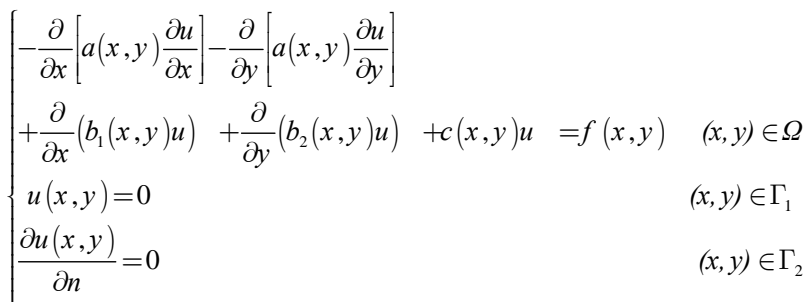

Of which area $\Omega=[0,1] \times[0,1], b_{1}(x, y)=2+y$,

$$
\begin{gathered}
b_{2}(x, y)=1+x, \\
c(x, y)=x+2 y, a(x, y)=1+2 x+3 y,
\end{gathered}
$$

True solution: $\mathrm{u}=x y(2-x)(1-y)$.

And $a(x, y)$ with $\alpha_{0}+\alpha_{1} x+\alpha_{2} y \quad$ as an approximation, the genetic algorithm parameter settings are as follows:

population size: 50;crossover probability: $P_{c}=0.8$;mutation probability $P_{m}=0.2$; termination of algebraic: 1000 .
Because of using $\alpha_{0}+\alpha_{1} x+\alpha_{2} y$ approximation $a(x, y)$, the Range of values of parameters $\alpha_{0}, \alpha_{1}, \alpha_{2}$ are unknown, First assume a default range of values and observe adapting the size of the value, then estimate the smaller range of parameters $\alpha_{0}, \alpha_{1}, \alpha_{2}$ exists in example 2 , set $-10 \leq \alpha_{i} \leq 10, \quad i=0,1,2$ after 1000times of calculating,

$$
\alpha_{0}=0.9359, \alpha_{1}=2.4119, \alpha_{2}=3.1738 \text {. }
$$

The mean square inaccuracy is 0.00081 , indicating the parameter value should be between 0 and 4 .so the parameter range is $0 \leq \alpha_{i} \leq 4, i=0,1,2$, after 3 times of calculation, the result graph 2 illustrates:

Graph 2 Calculation results

\begin{tabular}{|c|c|c|c|c|}
\hline \multicolumn{1}{c|}{$\alpha$} & $\alpha_{0}$ & $\alpha_{1}$ & $\alpha_{2}$ & mean \\
times & 1.1210 & 2.0011 & 2.7535 & $-1.6068 \times 10^{-6}$ \\
\hline 1 & 1.0859 & 2.0008 & 2.8247 & $-1.2100 \times 10^{-6}$ \\
\hline 2 & 1.0475 & 2.0005 & 2.9029 & $-7.1835 \times 10^{-7}$ \\
\hline 3 & 1 & 2 & 3 & \\
\hline exact & & & & \\
\hline solution & & & & \\
\hline
\end{tabular}

\section{CONCLUSION}

1.From the numerical simulation results and running time of example 1 we can see that when applying the finite element method in solving two-dimensional elliptic equation with variable coefficients, we can get a higher degree of accuracy, and speed is faster.

2.olving Two dimensional inverse problem is a very difficult task, the traditional method is either too complex in mathematical derivation or too heavy in Calculate work, which is difficult to apply to engineering practices. The approach of using nonlinear optimization method for two dimensional parameters inversion calculation of elliptic equations with variable coefficients, from the calculation results we can see the effectiveness and feasibility of it, which also provides a new, viable means to address similar issues.

\section{REFERENCES}

[1] Hou X L, Li H Y, Liu J. Optimal algorithm for minimization of maximum value problems and application of dynamic absorbor with multidof[J]. Journal of Vibration and Shock, 2008, 27(1): 100-103

[2] Deng Jian- guang , Yuan Hua-qiang Zhao Yue- long. Grid task scheduling strategy based on genetic- ant algorithm. Application Research of Computers,2011,28(12):4485-4499.

[3] Ma Wen-juan,Gao Feng.Image Reconstruction Method of Time Domain Fluorescence Diffuse Optical Tomography Based on the Third Order Simplified Spherical Harmonics 
[4] Liu Qing huai.Homotopy Method for Solving Noneonvex Optimization with Weak Quasi Normal Condition.acta mathematicae applicatae sinica, 2011,34(6):996-1006

[5] Ge W G. Boundary Value Problems in Nonlinear Ordinary Di®erential Equations[M]. Beijing: SciencePress, 2007.
[6] HOU Xiang-lin, QIAN Ying, WU Hai-tao.Optimization Algorithm of Boundary Value Problem for Nonlinear Ordinary Di®erential Equations. Chinese Journal of Engineering Mathematics, 2010, 27(4): 663-668 